\title{
Investigating the effect of captopril on reducing the severity of symptoms of acute respiratory distress syndrome in rats
}

\author{
Marjan Golshani ${ }^{1 \oplus}$, Mohammad Hossein Sanei ${ }^{\circledR}{ }^{\oplus}$, Maryam Jabbari ${ }^{3^{*} \oplus}$ \\ ${ }^{1}$ Associate Professor of Internal Medicine, Isfahan University of Medical Sciences, Isfahan, Iran \\ ${ }^{2}$ Associate Professor of Pathology, Isfahan University of Medical Sciences, Isfahan, Iran \\ ${ }^{3}$ Resident of Internal Medicine, Isfahan University of Medical Sciences, Isfahan, Iran
}

*Correspondence to

Maryam Jabbari; Email:

jabbari.mary@gmail.com

Received 14 Apr. 2021

Accepted 30 July 2021

Published online 11 Aug. 2021

Keywords: Captopril, Acute respiratory distress syndrome, Oleic acid

\begin{abstract}
Introduction: Acute respiratory distress syndrome (ARDS) occurs when fluid builds up in the tiny, elastic air sacs (alveoli) in the lungs.

Objectives: This study aimed to investigate the effect of captopril in reducing the symptoms of ARDS induced by oleic acid in experimental rats.

Materials and Methods: This experimental study was conducted on 14 Wistar male rats of 6-7 weeks weighing 200-250 g. In the first group, they received $1.25 \mathrm{mg} / \mathrm{kg}$ of captopril daily in glucose solution for one week, but the second group did not. After one week, these rats were anesthetized by $5 \%$ isoflurane respiration and oleic acid $(0.1 \mathrm{mg} / \mathrm{kg})$ was injected intraperitoneal for ARDS induction. During the first 6 hours after starting ARDS, the rats were randomly selected and after anesthesia with ether and autopsy, they were cut and the lung histology was conducted.

Results: Intensity of lung tissue edema in the group receiving captopril (Cap) was $2.83 \pm 0.41$ and in the captopril group with oleic acid (Cap+OA) was $2.50 \pm 0.55$. Additionally, $83.3 \%$ of the rats had severe pulmonary edema in the OA group which was statistically significant $(P=0.003)$. The "severity of inflammation and bleeding" in the $\mathrm{OA}+$ Cap group was significantly less than OA group $(P=0.026)$.

Conclusion: Captopril can play a significant role in reducing the damage of the lung tissue and severity of tissue edema and inflammation and bleeding. Therefore, it may be possible to use this drug in human samples with acute respiratory distress syndrome to prevent further damage.
\end{abstract}

\section{Introduction}

Citation: Golshani M Sanei $\mathrm{MH}$, Jabbari $\mathrm{M}$. Investigating the effect of captopril on reducing the severity of symptoms of acute respiratory distress syndrome in rats. Immunopathol Persa. 2022;8(1):e15. DOI:10.34172/ ipp. 2022.15
Acute respiratory distress syndrome (ARDS) is characterized by rapid progressive shortness of breath, tachypnea, and hypoxia. The pathophysiology of ARDS is not fully understood; however it is believed that ARDS occurs when a pulmonary injury or extra-pulmonary injury causes to release inflammatory mediators and thus stimulates neutrophil accumulation in the pulmonary microcirculation. Neutrophils damage the vascular endothelium and the alveolar epithelium, leading to pulmonary edema, the formation of the mucous membrane of the hyaline, the reduction of lung compulsions and the hardening of the air exchange (1).

In addition, high dose medications, injections of blood products, direct and indirect lung injuries such as trauma and burns have been reported as other causes of ARDS. It is estimated that $7.1 \%$ of all patients admitted to intensive care unit (ICU) and

\section{Key point}

The results of this study showed that captopril could play a significant role in reducing the damage of the lung tissue and severity of tissue edema and inflammation and bleeding.

$16.1 \%$ of all patients who use mechanical ventilation are ARDS (2).

The ARDS is associated with high mortality rates, which vary widely from $30 \%$ to $70 \%$ in several reports (3). Although the annual ARDS mortality rates have shown improvement in several studies $(4,5)$, ARDS remains a life-threatening disease with high mortality.

Treatment for ARDS is supportive and includes mechanical ventilation, stress ulcer prophylaxis and venous thromboembolism, nutritional support and treatment of underlying injury. The low flow volume, high end-of-expiratory pressure, and conservative

\footnotetext{
Copyright $\odot 2022$ The Author(s); Published by Nickan Research Institute. This is an open-access article distributed under the terms of the Creative Commons Attribution License (http://creativecommons.org/licenses/by/4.0), which permits unrestricted use, distribution, and reproduction in any medium, provided the original work is properly cited.
} 
fluid therapy may improve the outcome $(6,7)$.

Considering the importance of this disease and its associated risks, preventive measures against the onset of this disease have been investigated in various studies. ACE inhibitor or angiotensin-converting enzyme inhibitors (ACEI) drugs have been used in some studies related to animals in the prevention of $\operatorname{ARDS}(8,9)$.

In the body, the angiotensinogen, as a peptide hormone, is firstly produced by the liver and released in the blood. The angiotensinogen protein is converted to angiotensin I by the renin hormone and the angiotensin I is converted to angiotensin II by the ACE. Angiotensin II is a potent factor of vascular contraction (especially the hepatocytes), as well as various strategies (such as stimulating secretion of aldosterone and anti-cryoprotectant hormone) causing water and sodium retention. Therefore, the final function of angiotensin II hormone is to increase blood pressure, and the drugs in this group are used in hypertension treatment (9). From this category of drugs, captopril, in addition to treating hypertension, protects endothelial cells in the vasculature and improves their function (5) and by affecting some genes, reduces apoptosis in endothelial cells (4) and reduces serum levels of some inflammatory factors such as intercellular adhesion molecule-1 and coagulation factors such as von Willebrand and fibrinogen were reported in diabetic patients (10). The reninangiotensin system seems to play an important role in ARDS's pathophysiology. ACE and angiotensin II and its typ-1a receptor have improved ARDS, since ACE-2 and its type-2 receptor protect the severe pulmonary damage in the rats (10).

In an experimental study performed in rats, oleic acid was used as a toxic substance that induced ARDS and in the group that used captopril to prevent ARDS, lung injury and inflammatory factors were significantly lower than the control group (8). Captopril also reduced ARDS in rats injected with lipopolysaccharides (11), and ARDS caused by meconium aspiration in pigs (12). In a human study that was conducted on a number of patients who were hospitalized in ICU due to ARDS, it was found that in a group who had already received ACEI and continued during admission, the mortality rate was lower than the control group. However, other factors such as the duration of hospitalization in ICU and the duration of intubation or re-intubation in the two groups did not differ significantly.

\section{Objectives}

Due to limited human and animal studies regarding the effect of captopril on reducing the incidence and severity of ARDS-induced symptoms, this study aimed to investigate the effect of captopril in reducing the symptoms of ARDS induced by oleic acid in experimental rats.

\section{Materials and Methods}

The present study is an experimental study. In this study,
14 Wistar male rats weighing 200-250 grams and 6-7 weeks aged were studied. They were randomly divided into two equal groups of six for each, and two rats were considered as controls without any intervention. Both groups were kept in completely similar conditions for one week.

In the first group, the rats received $1.25 \mathrm{mg} / \mathrm{kg}$ of captopril daily in glucose solution for one week. The second group did not receive captopril during this week. Then, in both groups, after one week, these rats were anesthetized by $5 \%$ isoflurane respiration and the drug was performed through a jugular vein of cannulation. Oleic acid $(0.1 \mathrm{mg} /$ $\mathrm{kg}$ ) was injected intraperitoneal for ARDS induction (8).

In normal conditions, the animals studied had full access to water and food and they were kept at temperature of $64-79^{\circ} \mathrm{F}$. Then, during first six hours after starting ARDS, the rats were randomly selected and after anesthesia of the rats with ether and autopsy, a systematic method was used to cut them in certain areas and remove the lungs and any lesion was recorded on them. The lung histology was studied in this article. It should be noted that the lams were read by the pathologist, and since the grouping of the rats was based on the numbers, the reading of the lam was also random and the pathologist did not know which lam was related to the group.

The severity of lung involvement based on Berlin's pathology criteria (including intensity of lung tissue edema, intensity of inflammation and bleeding, mucous membrane hyaline, severity of damage to the alveolar epithelial cells) was evaluated and qualitatively scored mild, moderate and severe.

\section{Statistical analysis}

The collected data were entered into SPSS software (Version 22) and Fisher's exact test was used for evaluation of two groups and the significance level was less than 0.05 .

\section{Results}

The results of this study showed that the intensity of edema of the lung tissue in the group receiving captopril (Cap) was $2.83 \pm 0.41$ and the captopril group with oleic acid (OA+ Cap) was $2.50 \pm 0.55$ (50\% moderate and $50 \%$ severe). In addition, $83.3 \%$ of the rats had severe pulmonary edema in the group without receiving captopril, which was statistically significant $(P=0.003)$. In other words, in the OA group, due to lack of captopril (one week before induction of ARDS), the incidence of lung tissue edema was higher than the rats receiving captopril. In addition, the "severity of inflammation and bleeding" in the $\mathrm{OA}+\mathrm{Cap}$ group was significantly less than $\mathrm{OA}$ group $(P=0.026)$. In addition, although the "severity of epithelial alveolar cells lesions in the OA group was more than $\mathrm{OA}+\mathrm{Cap}$ group this difference was not statistically significant $(P=0.211)$. Finally, the formation of hyaline membrane in three groups was evaluated mild (Table 1 and Figure 1). 
Table 1. Comparison of frequency distribution of pulmonary involvement severity according to Berlin pathology criteria in the studied groups

\begin{tabular}{|c|c|c|c|c|c|}
\hline Berlin pathology criteria & & OA group $(n=6)$ & $O A+$ Cap $(n=6)$ & Control $(n=2)$ & $P$ value \\
\hline \multirow{3}{*}{ Severity of lung tissue edema } & Mild & $0(0.0 \%)$ & $0(0.0 \%)$ & $2(100.0 \%)$ & \multirow{3}{*}{0.003} \\
\hline & Moderate & $1(16.7 \%)$ & $3(50.0 \%)$ & $0(0.0 \%)$ & \\
\hline & Sever & $5(83.3 \%)$ & $3(50.0 \%)$ & $0(0.0 \%)$ & \\
\hline \multirow{3}{*}{ Severity of inflammation and bleeding } & Mild & $0(0.0 \%)$ & $2(33.3 \%)$ & $0(0.0 \%)$ & \multirow{3}{*}{0.026} \\
\hline & Moderate & $3(50.0 \%)$ & $4(66.7 \%)$ & $2(100.0 \%)$ & \\
\hline & Sever & $3(50.0 \%)$ & $0(0.0 \%)$ & $0(0.0 \%)$ & \\
\hline \multirow{3}{*}{ Hyaline membrane formation } & Mild & $6(100.0 \%)$ & $6(100.0 \%)$ & $2(100.0 \%)$ & \multirow{3}{*}{-} \\
\hline & Moderate & $0(0.0 \%)$ & $0(0.0 \%)$ & $0(0.0 \%)$ & \\
\hline & Sever & $0(0.0 \%)$ & $0(0.0 \%)$ & $0(0.0 \%)$ & \\
\hline \multirow{3}{*}{ Severity of alveolar epithelial cells' lesion } & Mild & $4(66.7 \%)$ & $5(83.3 \%)$ & $2(100.0 \%)$ & \multirow{3}{*}{0.211} \\
\hline & Moderate & $0(0.0 \%)$ & $1(16.7 \%)$ & $0(0.0 \%)$ & \\
\hline & Sever & $2(33.3 \%)$ & $0(0.0 \%)$ & $0(0.0 \%)$ & \\
\hline
\end{tabular}

OA: the oleic acid receptor group for induction ARDS.

OA+CAP: the captopril receptor group during one week then the oleic acid receptor for ARDS.

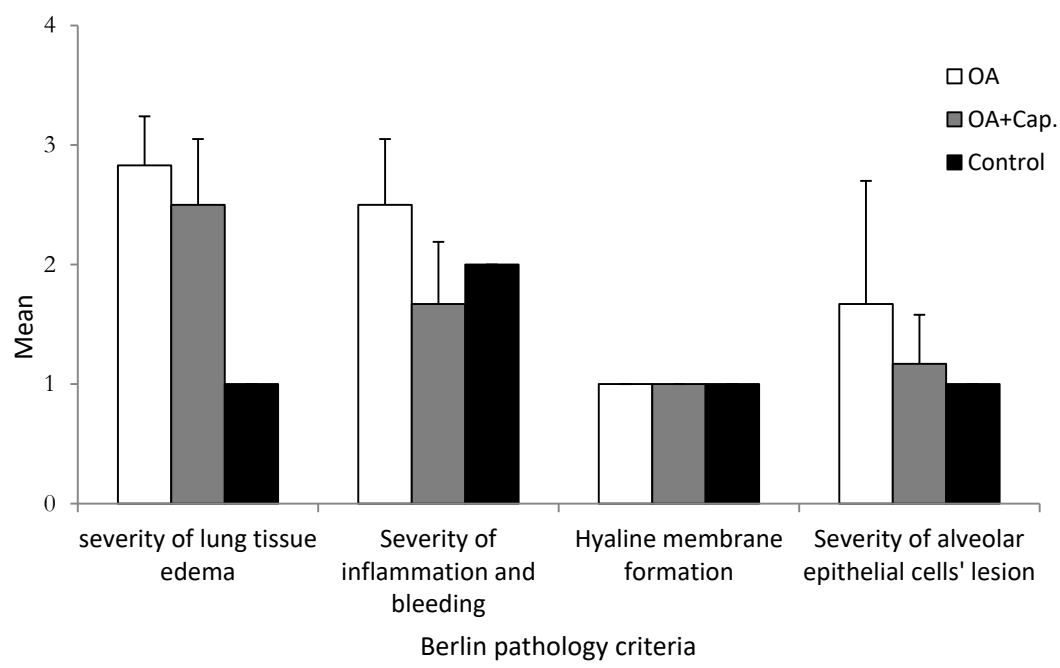

Figure 1. The mean of pulmonary involvement severity according to Berlin pathology criteria in the studied groups.

\section{Discussion}

ARDS as the most severe form of acute lung injury is one of the major challenges of intensive care medicine. At presenet, there is no specific therapy available although many efforts have been made in preventing and treating. It is still urgently needed to search for effective and specific therapies especially with conventional pharmaceutical drugs. Captopril is an orally active inhibitor of ACE which is widely used as an anti-hypertensive agent but it is known to modulate the neurohormonal system to reduce inflammation and preventing tissue fibrosis. However, the role of ACE inhibitor in the lungs is not well-understood (13). We, therefore, conducted this study to elucidate the severity of pulmonary involvement of oleic acid induced ARDS rats treated with captopril according to Berlin pathological criteria. Captopril therapy in rats with acute lung injury showed lower lung injury score or alveolar collagen that suggested inhibition of ACE could provide protective effects on acute lung injury (14). The mechanism may be the captopril attenuation of lung fibrosis in lung epithelial cells by abrogating apoptosis. Numerous in vitro studies have shown the relation of angiotensin II as the final product of ACE activity, to production of human lung fibroblast collagen in a bleomycin-toxicity model. This was weakened by ACE inhibitor or angiotensin receptor blocker therapy (15). Similarly, inhibition of the renin-angiotensin aldosterone axis has been considered as a potential target for ARDS prevention and showed encouraging preclinical data. Captopril as an angiotensinconverting enzyme inhibitor has some effects such as reducing degradation of bradykinin and inhibition of angiotensin synthesis. It can also lower the pulmonary arterial hypertension and prevent pathological refactoring of cardiovascular. Captopril can obviously do the screening of oxygen free radicals, slow lipid peroxidation, provide the recovery of pulmonary vascular endothelial dysfunction, reduce vascular permeability, attenuate tissue edema and inflammatory exudation, and revealed 
certain anti-inflammatory and anti-fibrosis effects (13). In a study on the rat model of chemically induced ARDS, captopril therapy improved several substitute markers of severe ARDS such as circulating endothelial cells, partial pressure of oxygen $(\mathrm{PaO} 2)$, and wet to dry lung weight ratio (16). In another model of ARDS rats subjected to ventilator-induced lung injury, markers of inflammation and apoptosis significantly reduced in captopril treated versus in untreated controls (17). In a similar design, Yao et al showed losartan can be effective at mitigating the lung injury induced ventilator in rats (18).

The results of this study showed that although the level of hyaline membrane formation and the severity of alveolar epithelial cell damage did not differ significantly between the two groups, the severity of lung edema in the captopril group was significantly lower than the one receiving the oleic acid alone; In the OA+Cap group, 50\% of the rats had severe pulmonary edema, whereas in the OA group, more than $80 \%$ of the mice had severe lung edema, so it can be said that captopril has been able to prevent the edema of the lung tissue.

In a study by Xiaolin et al, a non-treated OA group of acute lung injury rats was compared with a captopril treated group and showed the use of captopril prevented the symptoms of OA-induced severe lungs injury in rats with a significant lower lung injury score, less albumin content and alveolar infiltrated cells, decreased wet/ dry weight ratio of the lung tissues, and improved lung function ( $\mathrm{PaO} 2$ per fraction of inspired oxygen). Captopril also considerably reduced the expression of intercellular adhesion molecule- 1 in the lung tissue and in blood circulating endothelial cells that indicate its protective effect on these cells activation or damage (8). Accordingly, our data suggested that ACE inhibition with clinical use of inhibitor offers protective effects on ARDS and potential administration for therapeutic option. The results of the current study also showed the incidence of lung tissue edema was higher in the OA group than the rats receiving captopril. In addition, the "severity of inflammation and bleeding" in the OA+Cap group was significantly less than OA group. Typical OA induced lung injury is featured with thickening of the alveolar septa, alveolar hemorrhage, and infiltration of inflammatory cells due to the direct damage of the pulmonary endothelium. All these features were observed in the lung tissue of captopril treated animals and it significantly reduced the lung injury with less interstitial edema, hemorrhage, and cellular infiltration.

Knowing about the role of ACE on ARDS and the related endothelial cell damage opens a new window for pharmacological approaches. Although the inhibition and/or the elimination of ACE with molecular biological and transgenic techniques showed beneficial effects on animal models (19), this is far from clinical application. On the other hand, pharmaceutical use of ACE inhibitors such as captopril has been successfully conducted in clinical as antihypertension drugs but have not yet been tested for respiratory syndrome. Our data showed that the administration of captopril after OA challenge significantly reduced the severity of lung injury and improved the blood oxygenation in the rats. The novelty demonstrated in this study is that early administration of a pharmaceutical ACE inhibitor may be able to prevent animals from severe ARDS. The results of this study have also been shown that the severity of inflammation and bleeding in the captopril group was less than the group of OA alone. In other words, in the OA+Cap group no mice had severe inflammation and bleeding, while in the OA group, 50\% of the mice had severe inflammation and bleeding. The OA-induced ARDS is known as a very severe model (20) and the beneficial effects of captopril in this model have strongly implicated its potential therapeutic effects in such pathological conditions. Considering the lack of specific therapies ARDS, using the conventional ACE inhibitors as a potential treatment is obviously an attractive and practical strategy deserved to be further studied.

One of the weaknesses of the present study was the lack of repeated follow-up of lung tissue at 1, 3, 6 and 12 hours after intervention, which was not possible due to the small sample size, and according to previous studies there was no significant difference in time over these intervals. Nevertheless, it is suggested that this evaluation be repeated in future studies.

\section{Conclusion}

According to the results of this study, the administration of captopril can play a significant role in reducing the damage of the lung tissue due to the severity of tissue edema and inflammation and bleeding. Therefore, it may be possible to use this drug in human samples with ARDS to prevent further damage to the lung tissue.

\section{Authors' contribution}

MG, MHS and MJ were the principal investigators of the study. MJ participated in preparing the concept and design. MG and MHS critically evaluated the intellectual contents. All authors participated in preparing the final draft of the manuscript, revised the manuscript and critically evaluated the intellectual contents. All authors have read and approved the content of the manuscript and confirmed the accuracy or integrity of any part of the work.

Conflicts of interest

The authors declare that they have no competing interests.

\section{Ethical issues}

This study was confirmed by Local Ethics Committee of Isfahan University of Medical Sciences (Code\#IR.MUI.REC.1396.3.981), based on the ethical principles of the International Committees for the Protection of Animal Rights Laboratory and National Health and Medical Research Council guidelines. Prior to the trial, the protocols like animal care, nutrition and prescribing method, anesthesia and euthanasia procedures were approved to be in agreement with the rules of the ethical committee of this university. This study was extracted from internal medicine residential thesis of Maryam Jabbari at this university (Thesis \# 396981). Additionally, ethical 
issues (including plagiarism, data fabrication, double publication) have been completely observed by the authors.

\section{Funding/Support}

This work supported by deputy research and technology of Isfahan University of Medical Sciences (Grant\# 396981).

\section{References}

1. Matthay MA, Zemans RL. The acute respiratory distress syndrome: pathogenesis and treatment. Annu Rev Pathol. 2011;6:147-63. doi:10.1146/annurev-pathol-011110-130158.

2. Frutos-Vivar F, Nin N, Esteban A. Epidemiology of acute lung injury and acute respiratory distress syndrome. Curr Opin Crit Care. 2004;10:1-6. doi: 10.1097/00075198-20040200000001.

3. Bannerman DD, Goldblum SE. Mechanisms of bacterial lipopolysaccharide-induced endothelial apoptosis. Am J Physiol Lung Cell Mol Physiol. 2003;284:L899-914. doi: 10.1152/ajplung.00338.2002

4. Hamdi HK, Castellon R. ACE inhibition actively promotes cell survival by altering gene expression. Biochem Biophys Res Commun. 2003;310:1227-35. doi: 10.1016/j. bbrc.2003.09.149.

5. Fleming I, Kohlstedt K, Busse R. New fACEs to the reninangiotensin system. Physiology. 2005;20:91-5. doi: 10.1152/ physiol.00003.2005.

6. Krag M, Perner A, Wetterslev J, Wise MP, Møller MH. Stress ulcer prophylaxis versus placebo or no prophylaxis in critically ill patients. Intensive Care Med. 2014;40:11-22. doi: 10.1007/ s00134-013-3125-3.

7. Lin PC, Chang $\mathrm{CH}$, Hsu PI, Tseng PL, Huang YB. The efficacy and safety of proton pump inhibitors vs histamine-2 receptor antagonists for stress ulcer bleeding prophylaxis among critical care patients: a meta-analysis. Crit Care Med. 2010;38:1197205. doi: 10.1097/CCM.0b013e3181d69ccf.

8. He X, Han B, Mura M, Xia S, Wang S, Ma T, et al. Angiotensinconverting enzyme inhibitor captopril prevents oleic acidinduced severe acute lung injury in rats. Shock. 2007;28:10611. doi: 10.1097/SHK.0b013e3180310f3a.

9. Yu QH, Guo JF, Chen Y, Guo XR, Du YQ, Li ZS. Captopril pretreatment protects the lung against severe acute pancreatitis induced injury via inhibiting angiotensin II production and suppressing Rho/ROCK pathway. Kaohsiung J Med Sci. 2016;32:439-45. doi: 10.1016/j.kjms.2016.07.008.

10. Rosei EA, Rizzoni D, Muiesan ML, Sleiman I, Salvetti M, Monteduro C, et al. Effects of candesartan cilexetil and enalapril on inflammatory markers of atherosclerosis in hypertensive patients with non-insulin-dependent diabetes mellitus. Hypertens J. 2005;23:435-44. doi: 10.1097/00004872200502000-00027.

11. Li Y, Zeng Z, Cao Y, Liu Y, Ping F, Liang M, et al. Angiotensinconverting enzyme 2 prevents lipopolysaccharide-induced rat acute lung injury via suppressing the ERK $1 / 2$ and NF-KB signaling pathways. Sci Rep. 2016;6:27911. doi: 10.1038/ srep27911.

12. Treml B, Loeckinger A, Kleinsasser A, Schoepf E, Geiger R, Neu N. Angiotensin-Converting Enzyme 2 Improves Arterial Hypoxemia in Meconium-Induced Acute Lung Injury in Piglets. Adv Crit Care. 2015;2015. doi: 10.1155/2015/495675.

13. Liu HM, Guo YN. Effect of captopril on serum TNF- $\alpha$ level in acute lung injury rats induced by HCL. Asian Pac J Trop Med. 2014;7:905-8. doi: 10.1016/S1995-7645(14)60158-9.

14. Jerng JS, Hsu YC, Wu HD, Pan HZ, Wang HC, Shun CT, et al Role of the renin-angiotensin system in ventilator-induced lung injury: an in vivo study in a rat model. Thorax. 2007;62:527535. doi: 10.1136/thx.2006.061945.

15. Marshall RP, Gohlke P, Chambers RC, Howell DC, Bottoms SE, Unger T, et al. Angiotensin II and the fibroproliferative response to acute lung injury. Am J Physiol Lung Cell Mol Physiol. 2004;286:L156-64. doi: 10.1152/ajplung.00313.2002.

16. Liu H, Zhao J. An experimental study of therapeutic effect of ACEI on chemical-induced ARDS in rats. Zhonghua Yu Fang Yi Xue Za Zhi. 2002;36:93-6.

17. Wösten-van Asperen RM, Lutter R, Haitsma JJ, Merkus MP, van Woensel JB, Van Der Loos CM, et al. ACE mediates ventilator-induced lung injury in rats via angiotensin II but not bradykinin. Eur Respir J. 2008;31:363-71. doi: 10.1183/09031936.00060207.

18. Reddy R, Asante I, Liu S, Parikh P, Liebler J, Borok Z, et al. Circulating angiotensin peptides levels in Acute Respiratory Distress Syndrome correlate with clinical outcomes: A pilot study. PLoS One. 2019;14:e0213096. doi: 10.1371/journal. pone.0213096.

19. Wohlrab P, Kraft F, Tretter V, Ullrich R, Markstaller K, Klein KU. Recent advances in understanding acute respiratory distress syndrome. F1000Res. 2018;7:F1000 Faculty Rev-263. doi: 10.12688/f1000research.11148.1

20. Laffey JG, Bellani G, Pham T, Fan E, Madotto F, Bajwa EK, et al. Potentially modifiable factors contributing to outcome from acute respiratory distress syndrome: the LUNG SAFE study. Intensive Care Med. 2016;42:1865-76. doi: 10.1007/ s00134-016-4571-5. 